Case Report

\title{
An Uncommon Cause of a Small-Bowel Obstruction
}

\author{
Ali Zakaria, Bayan Al Share, Issam Turk, Samira Ahsan, and Waseem Farra \\ Department of Internal Medicine, Division of Pulmonology, Providence-Providence Park Hospital, \\ Michigan State University College of Human Medicine, Southfield, MI, USA \\ Correspondence should be addressed to Ali Zakaria; alizakaria86@hotmail.com \\ Received 17 February 2017; Accepted 5 March 2017; Published 12 March 2017 \\ Academic Editor: Shinji Tanaka \\ Copyright (C) 2017 Ali Zakaria et al. This is an open access article distributed under the Creative Commons Attribution License, \\ which permits unrestricted use, distribution, and reproduction in any medium, provided the original work is properly cited.

\begin{abstract}
Sarcoidosis is a systemic granulomatous disease of unknown etiology, characterized by the formation of noncaseating granulomas. Gastrointestinal (GI) system involvement that is clinically recognizable occurs in less than $0.9 \%$ of patients with sarcoidosis, with data revealing small intestine involvement in $0.03 \%$ of the cases. A high index of suspension is required in patients presenting with small-bowel obstruction and previous history of sarcoidosis. Establishing a definitive diagnosis of GI sarcoidosis depends on biopsy evidence of noncaseating granulomas, exclusion of other causes of granulomatous disease, and evidence of sarcoidosis in at least one other organ system. Treatment of GI sarcoidosis depends on symptomatology and disease activity. Herein, we are presenting a case of 67-year-old female patient who had acute small-bowel obstruction at the level of jejunum with postoperative histopathologic evidence of noncaseating granulomatous inflammation with multinucleated giant cells, consistent with sarcoidosis.
\end{abstract}

\section{Introduction}

Sarcoidosis is a systemic granulomatous disease of unknown etiology, characterized by the formation of noncaseating granulomas. Gastrointestinal (GI) involvement is very rare and can occur in a patient with known sarcoidosis or as the initial manifestation of the disease. Small-bowel obstruction secondary to sarcoidosis has been described in few case reports. Herein, we are reporting a case of 67-year-old female patient who had acute small-bowel obstruction at the level of jejunum with postoperative histopathologic evidence of noncaseating granulomatous inflammation consistent with sarcoidosis.

\section{Case Report}

A 67-year-old female with previous medical history of inactive pulmonary sarcoidosis (Figure 1), hypertension, and impaired glucose tolerance, presented to emergency department with five-day history of nausea, vomiting, abdominal pain, and constipation. On physical examination she was alert, oriented, and not in acute distress. Her vital signs were as follows: Temp $36.7^{\circ} \mathrm{C}$; pulse $93 \mathrm{bpm}$; respiratory rate $18 \mathrm{bpm}$; blood pressure $147 / 85 \mathrm{mmHg}$; and $\mathrm{O}_{2} \%$ of $98 \%$ on room air. Her abdomen was distended and mildly tender over the epigastric area, with no evidence of organomegaly. Her bowel sounds were hyperactive. Initial imagining studies with abdominal X-ray revealed dilated small-bowel loops with multiple scattered air fluid levels suggesting partial versus early complete small-bowel obstruction. A contrasted computed tomography (CT) scan of the abdomen and pelvis demonstrated multiple dilated loops of proximal small bowel with no definitive transition point and no intraperitoneal free air or fluid identified, a picture suggestive of a high-grade small-bowel obstruction (Figure 2).

She was managed conservatively with bowel rest, nasogastric tube, and IV fluids. Her symptoms failed to improve, so gastrografin small-bowel follow-through was performed and demonstrated failure of contrast to pass into the colon (Figure 3).

General surgery was consulted and diagnostic laparoscopy was performed and revealed multiple dilated bowel loops which revealed diffuse skin tag appearing lesions on the antimesenteric surface of the midjejunum causing inflammatory adhesions to the mesentery (Figure 4). The bowel was completely viable with no additional lesions in the liver, stomach, or visible parts of the colon. Histopathologic examination of tissue biopsy revealed lymphohistiocytic noncaseating granulomatous inflammation with multinucleated giant cells, consistent with sarcoidosis (Figure 5). Further 

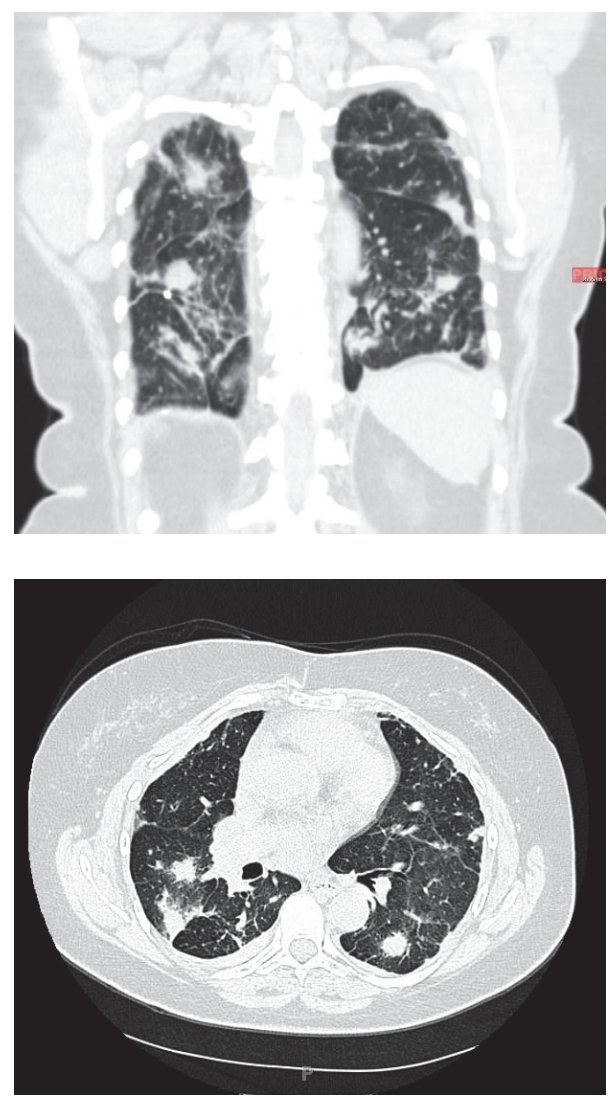

FIGURE 1: Computed tomography (CT) scan of the chest reveals diffuse parenchymal nodularity, with no honeycombing, hilar, or mediastinal adenopathy, consistent with stage III sarcoidosis.

evaluation revealed negative acid-fast bacilli and fungal and viral tissue cultures. Her postoperative course was uncomplicated and she was discharged on prednisone taper dose with close outpatient follow-up.

\section{Discussion}

Sarcoidosis is a systemic granulomatous disease of unknown etiology, characterized by the formation of noncaseating granulomas. Gastrointestinal (GI) system involvement that is clinically recognizable occurs in $0.1-0.9 \%$ of patients with sarcoidosis, with one study revealing postmortem small intestine involvement on autopsy in $0.03 \%$ of the cases $[1,2]$. It usually occurs in patients in their fifth or sixth decade of life with evidence of multisystem sarcoidosis in approximately one-half of patients. The most common manifestation is nonbloody diarrhea with colicky abdominal pain. It can also present with nonspecific constitutional symptoms, obstruction, and/or intestinal hemorrhage, a presentation that can mimic other diseases such as Crohn's disease. Other rare manifestations include mesenteric venous insufficiency due to pressure from enlarged sarcoid lymphadenopathy, megaloblastic anemia caused by terminal ilium infiltration, and protein-losing enteropathy [3-6]. Establishing a definitive
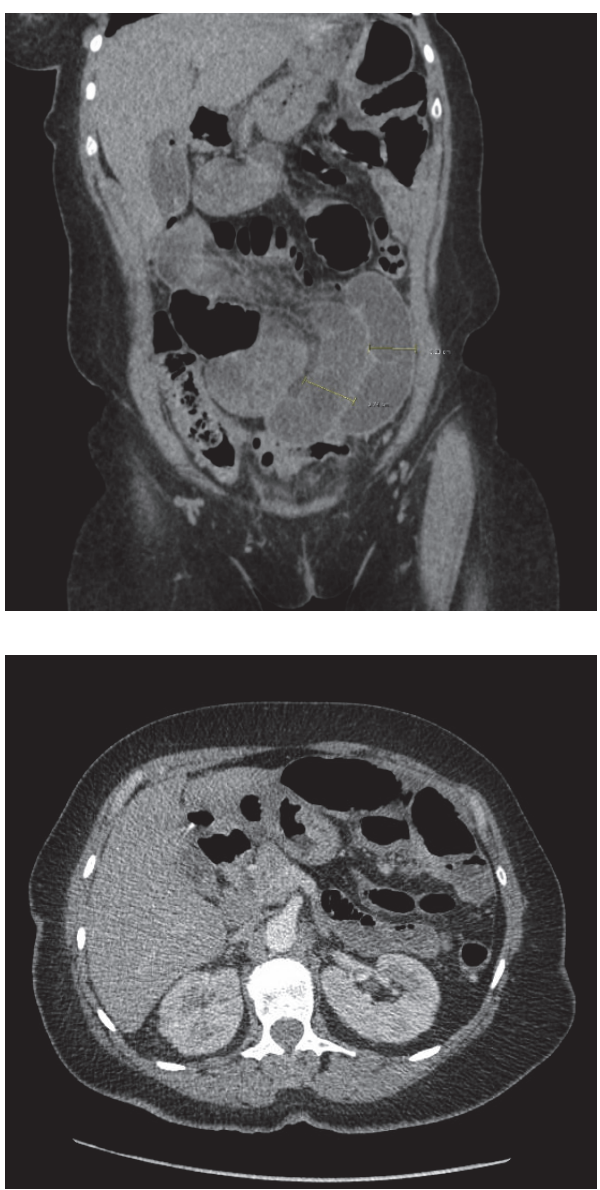

Figure 2: Computed tomography (CT) scan of the abdomen demonstrated early complete bowel obstruction at the level of midjejunum.

diagnosis of GI sarcoidosis depends on three components: (1) biopsy evidence of noncaseating granulomas in the symptomatic or incident organ, (2) exclusion of other causes of granulomatous disease, particularly mycobacterial, fungal, and parasitic infections, and (3) clinical, radiographic, and optimally histopathologic evidence of sarcoidosis in at least one other organ system [7]. Imaging studies using computed tomography scan considered appropriate initial step in diagnosing patients who present with abdominal pain, while, in those who present with prominent diarrhea, endoscopy is more appropriate. Treatment of GI sarcoidosis depends on symptomatology and disease activity; asymptomatic patients can be monitored without active therapy, while symptomatic patients with substantial organ involvement and granulomatous inflammation on tissue biopsy should be treated with corticosteroid for six to eight weeks or until a response to therapy is noted and then gradually tapered, over a period of approximately six months [7]. The optimal duration of corticosteroid treatment for gastrointestinal sarcoidosis is not known, but experts recommend treating the initial manifestation for at least one year. Other treatments such as surgical intervention may be necessary in patients with bowel 


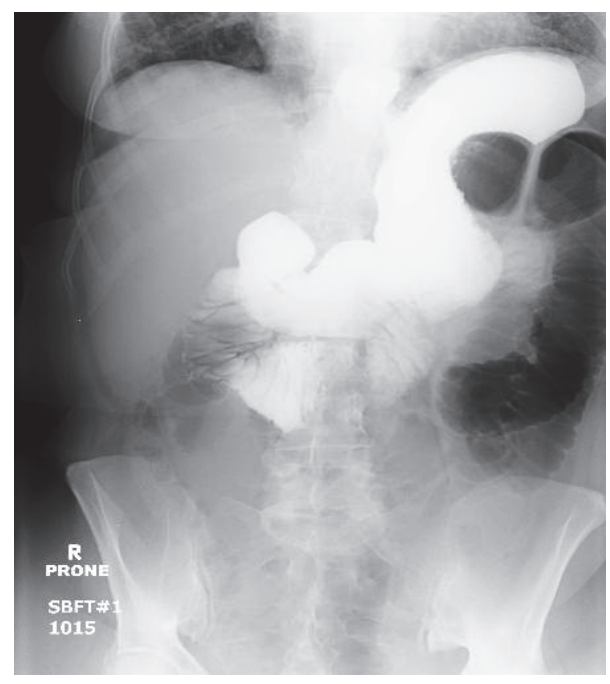

FIGURE 3: Gastrografin small-bowel follow-through demonstrated failure of contrast to pass into the colon.

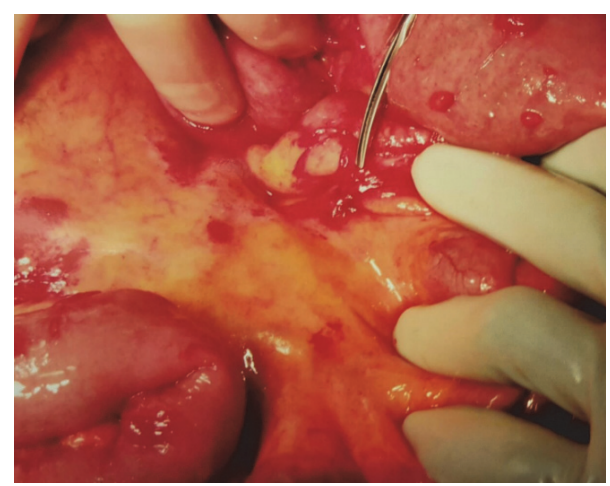

FIGURE 4: Intraoperative demonstration of the skin tag appearing lesion at the level of midjejunum causing inflammatory adhesions.

obstruction, perforation, or massive hemorrhage. Monitoring of GI sarcoidosis is achieved clinically and radiographically, but there is no evidence for the role of serum angiotensin converting enzyme or serum interlukin-2 receptor in monitoring $[8,9]$.

\section{Conclusion}

Gastrointestinal (GI) sarcoidosis is very rare and can occur in a patient with known diagnosis or as initial presentation. A high index of suspension is required in patients presenting with small-bowel obstruction and previous history of sarcoidosis.

\section{Conflicts of Interest}

The authors declare that there are no conflicts of interest regarding the publication of this paper.

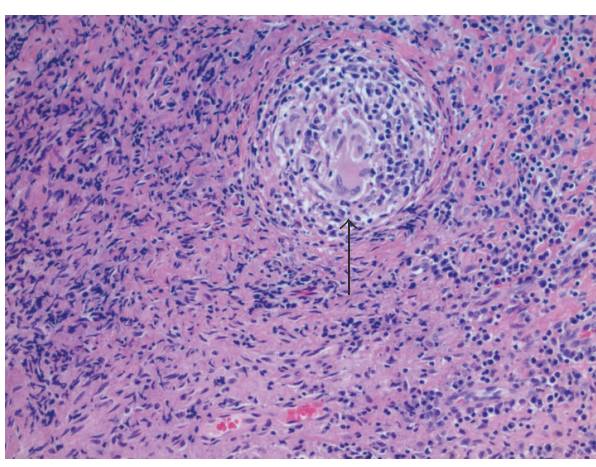

(a)

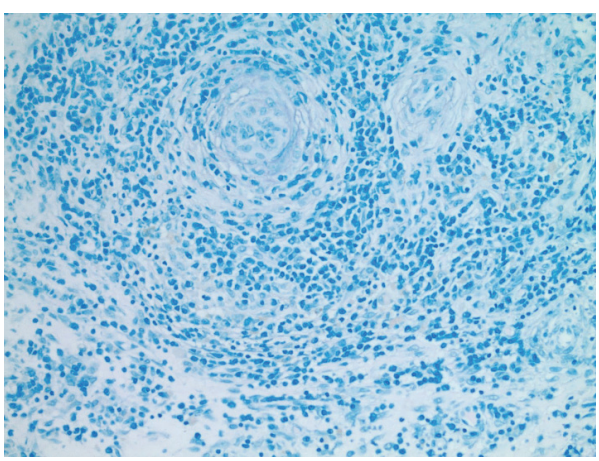

(b)

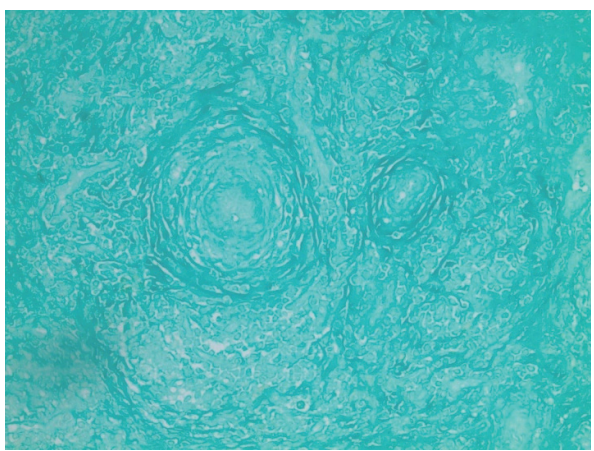

(c)

FIGURE 5: Histopathology of the resected small-bowel lesion. (a) H\&E stain revealed lymphohistiocytic noncaseating granuloma with multinucleated giant cells (black arrow). (b) Negative for acidfast bacilli (AFB) stain. (c) GMS stain negative for fungal infection.

\section{Acknowledgments}

Authors would like to express their deepest appreciation to Dr. Juanita Evans for her valuable expertise in providing and reading the histopathology slides.

\section{References}

[1] A. M. Sharma, J. Kadakia, and O. P. Sharma, "Gastrointestinal sarcoidosis," Seminars in Respiratory Medicine, vol. 13, no. 6, pp. 442-449, 1992.

[2] K. Iwai, T. Takemura, M. Kitaichi, Y. Kawabata, and Y. Matsui, "Pathological studies on sarcoidosis autopsy. II. Early change, 
mode of progression and death pattern," Acta Pathologica Japonica, vol. 43, no. 7-8, pp. 377-385, 1993.

[3] W. Schwarzschild and R. M. Myerson, "Venous insufficiency of the small intestine secondary to sarcoidosis of mesenteric lymph nodes," American Journal of Gastroenterology, vol. 50, no. 1, pp. 69-72, 1968.

[4] R. H. Fleming, M. Nuzek, and D. W. McFadden, "Small intestinal sarcoidosis with massive hemorrhage: report of a case," Surgery, vol. 115, no. 1, pp. 127-131, 1994.

[5] A. Rauf, P. Davis, and H. Levendoglu, "Sarcoidosis of the small intestine," American Journal of Gastroenterology, vol. 83, no. 2, pp. 187-189, 1988.

[6] O. S. Popović, S. Brkić, P. Bojić et al., "Sarcoidosis and protein losing enteropathy," Gastroenterology, vol. 78, pp. 119-125, 1980.

[7] E. C. Ebert, M. Kierson, and K. D. Hagspiel, "Gastrointestinal and hepatic manifestations of sarcoidosis," The American Journal of Gastroenterology, vol. 103, no. 12, pp. 3184-3192, 2008.

[8] J. C. Grutters, J.-M. Fellrath, L. Mulder, R. Janssen, J. M. M. Van Den Bosch, and H. Van Velzen-Blad, "Serum soluble interleukin-2 receptor measurement in patients with sarcoidosis: a clinical evaluation," Chest, vol. 124, no. 1, pp. 186-195, 2003.

[9] S. Miyoshi, H. Hamada, T. Kadowaki et al., "Comparative evaluation of serum markers in pulmonary sarcoidosis," Chest, vol. 137, no. 6, pp. 1391-1397, 2010. 


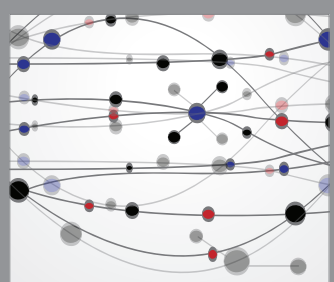

The Scientific World Journal
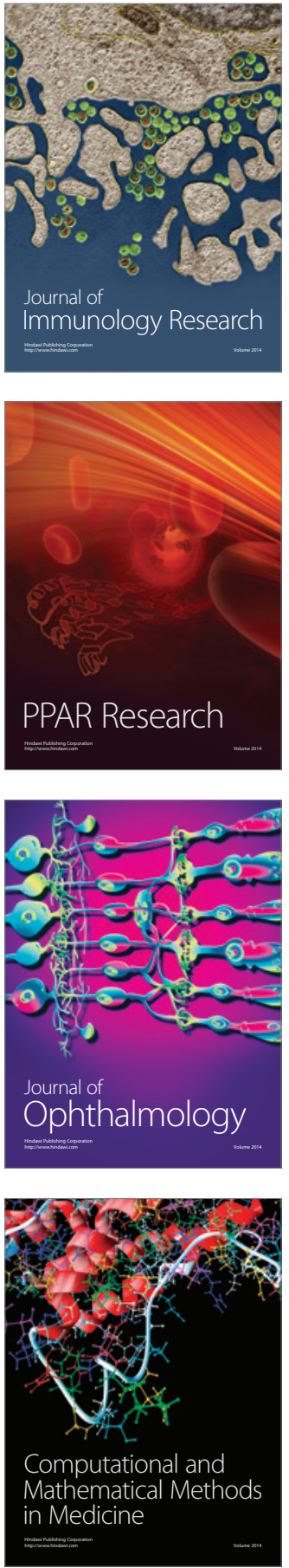

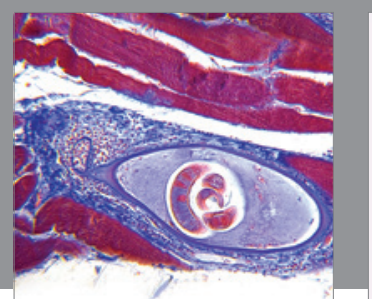

Gastroenterology Research and Practice
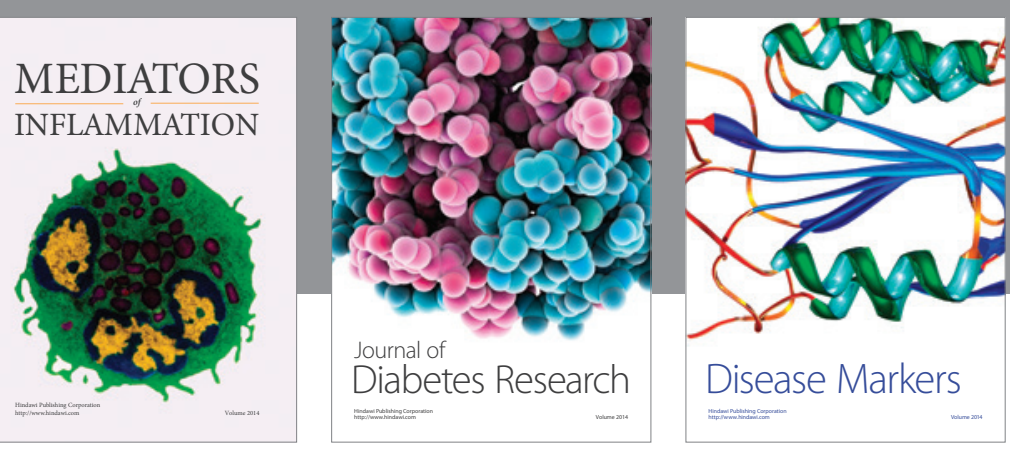

Disease Markers

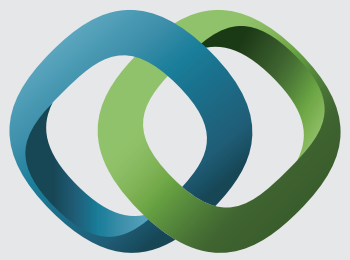

\section{Hindawi}

Submit your manuscripts at

https://www.hindawi.com
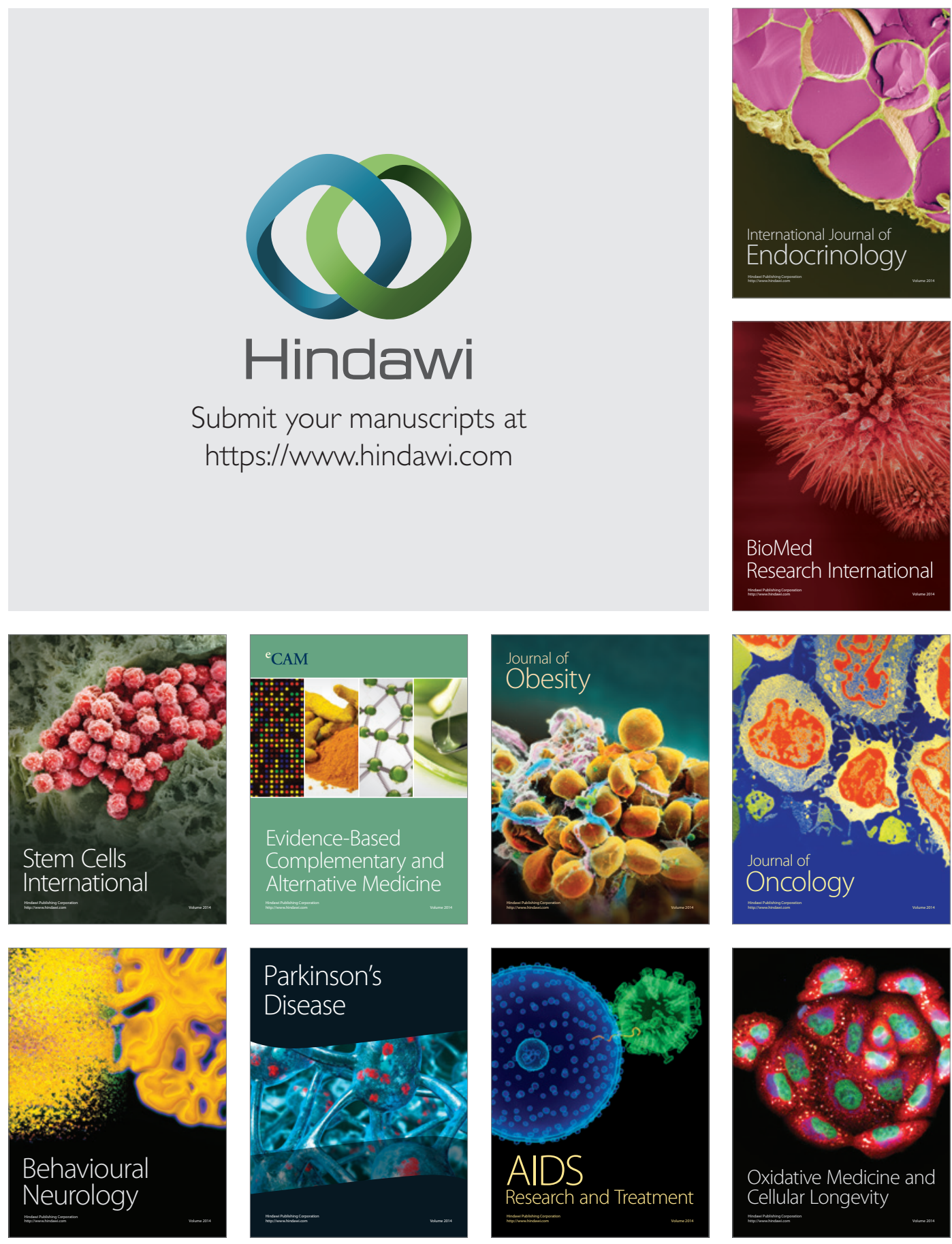